INFECTION

\section{Plazomicin once daily effective for complicated UTI}

The aminoglycoside plazomicin is noninferior to meropenem for treating complicated urinary tract infections (cUTIs), according to a study in The New England Journal of Medicine.

Previous treatment standards, such as fluoroquinolones and cephalosporins, are becoming less effective owing to increased resistance, which has led to an increase in the use of carbapenems (such as meropenem) for multidrug-resistant infections. However, the rise of carbapenem-resistant infections means alternative treatment options are now required.

The multinational, randomized, double-blind, phase III trial was designed to determine noninferiority of plazomicin over meropenem for cUTI. Eligible patients $(n=609)$ were randomized 1:1 to receive plazomicin $15 \mathrm{mg} / \mathrm{kg}$ daily or meropenem (1 g 8-hourly) for $\geq 4$ days of intravenous therapy, after which oral step-down therapy could begin. Primary end points were composite cure (clinical cure and microbiological eradication) at day 5 and at the test-of-cure visit carried out at days 15-19.

Composite cure was noted in $88.0 \%$ of patients who received plazomicin, compared with $91.4 \%$ of meropenem-treated patients at day 5 . who received plazomicin remained infection-free, compared with $70.1 \%$ of those who received meropenem, and more plazomicin-treated patients demonstrated microbiological eradication than meropenemtreated patients $(78.8 \%$ and $68.6 \%$, respectively). At a late follow-up point (24-32 days), fewer patients who had received plazomicin experienced microbiological recurrence. At test-of-cure, $81.7 \%$ of patients

"This is the first robust study showing that aminoglycoside monotherapy with plazomicin is effective in cUTI and/or pyelonephritis and can be a treatment alternative in this indication," Wagenlehner tells Nature Reviews Urology.

The focus on aminoglycosides also

fewer patients who had

received

plazomicin experienced microbiological recurrence

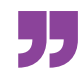

\title{
FROM THE MEETING
}

\section{Barcelona! What a beautiful (therapeutic) horizon}

\footnotetext{
The world of urology has been focused on Barcelona, which played host to the 34th European Association of Urology (EAU) congress.

Day 1 of the congress included the Opening Ceremony, which featured excerpts from Bizet's Carmen, setting the tone for an exciting meeting. Prizes were awarded to Freddie Hamdy, who was the recipient of the Willy Gregoir Medal, and Maarten Albersen, who received the coveted Crystal Matula award.

The plenary session kicked off day 2 with a discussion about bladder cancer in the young female patient. Fiona Burkhard chaired the session, commenting on the fact that $1-2 \%$ of patients will be $<40$ years of age. The updated EAU Guidelines were also released, resulting in a long line of delegates snaking through the exhibition hall to collect their copies. Ever-popular, the Guidelines Controversies sessions included
}

a debate on the role of prebiopsy MRI for prostate cancer. The debate was led by Caroline Moore, who argued that the adverse effects of biopsy can be significant and unnecessary in men with a negative MRI, and Sigrid Carlsson, who raised concerns regarding the accuracy and interobserver variability of MRI and instead suggested alternative treatment pathways. Importantly, the patient's viewpoint was emphasized, with a push to include patients in shared decision-making in this situation. A particularly exciting report from the meeting was the presentation of

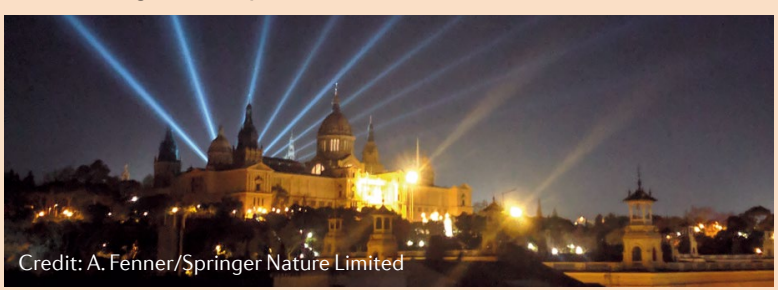

illustrates a change in attitudes towards these drugs: "Aminoglycosides have been infrequently recommended in the past 20 years, owing to their presumed toxicity," explains Wagenlehner. "In this study there was no dramatic increase in toxicity: a somewhat higher but still low creatinine increase compared with meropenem in patients with reduced creatinine clearance, but no ototoxicity." Furthermore, these data are also relevant in the context of antimicrobial stewardship, as aminoglycosides exert less collateral damage to the microbiome than carbapenems, cephalosporins or fluoroquinolones.

Annette Fenner

ORIGINAL ARTICLE Wagenlehner, F. M. E. et al. Once-daily plazomicin for complicated urinary tract infections. N. Engl. J. Med. https://doi.org/ 10.1056/NEJMoa1801467 (2019)

the largest ever study of testosterone replacement in men with prostate cancer, showing that this approach might actually slow tumour recurrence and calling into question the dogma of testosterone being protumorigenic. The team from the University of California Irvine, led by Thomas Ahlering, showed that tumour recurrence occurred in only $\sim 5 \%$ of treated patients, whereas the cancer had recurred in $15 \%$ of the patients who did not receive testosterone.

Sunday evening included the always popular BJUI Social media awards, now in its seventh year. Awardees included \#RudeFood for the Best Social Media Campaign, @Uropoet for Social Media Creativity and the late Daniel Christidis, who was posthumously awarded the Most Social Trainee Award. The big award of the night Outstanding Contribution to Social Media in Urology - was awarded to us here at Nature Reviews Urology for 'Both sides of the scalpel'.

As always, an excellent scientific and social programme proved a great success for EAU, and we look forward to next year's meeting.

Annette Fenner 